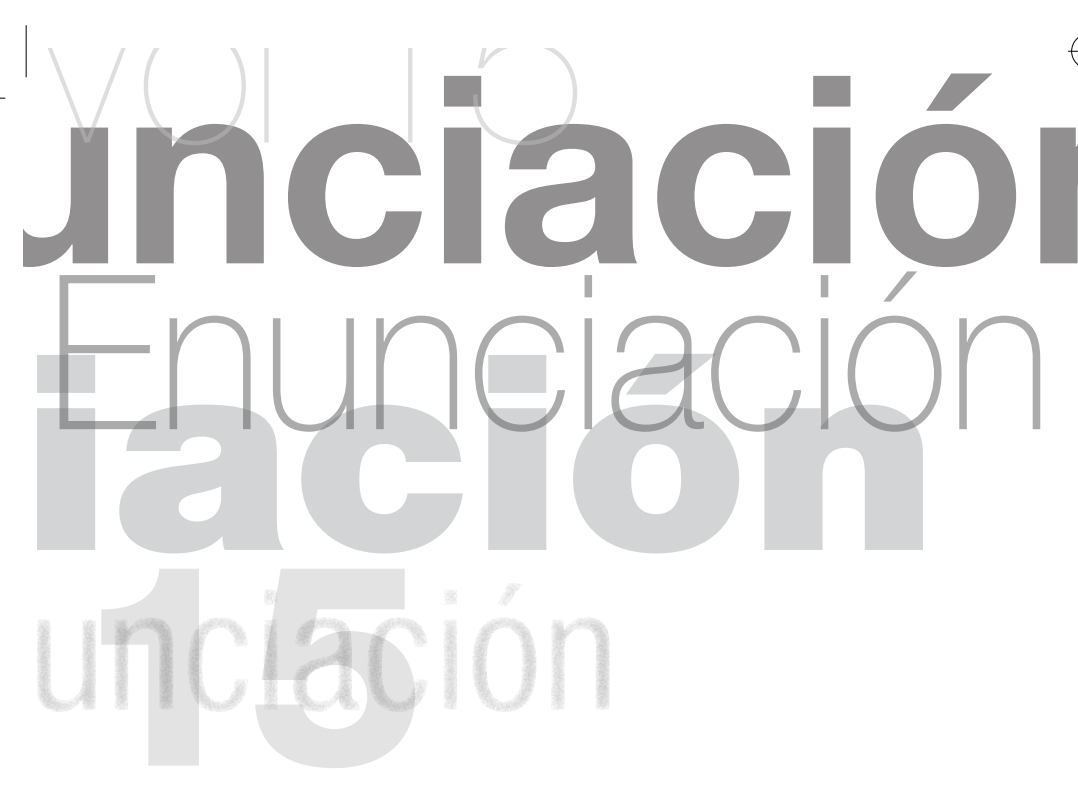

\title{
Lectura y escritura en la universidad: las consignas de tareas en la planificación de la reenseñanza de la lengua*
}

\author{
Reading and writing at \\ university: the instructions of \\ procedures in the organization of re- \\ teaching language
}

\begin{abstract}
Dora Riestra**
Al igual que un molde da forma a una sustancia, las palabras pueden transformar una actividad en una estructura. No obstante, dicha estructura puede ser modificada o remodelada cuando los niños aprenden a utilizar el lenguaje de modo que les permita ir más allá de las experiencias precedentes al planear una acción futura. El desarrollo de los procesos psicológicos superiores. Lev Vygotski. (1996)
\end{abstract}

\section{Resumen:}

Se trata de una propuesta didáctica de reenseñanza de la lengua formulada para el primer año del nivel universitario. Parte del enfoque histórico-cultural (Vygotski, Leontiev, Bajtín, Bronckart) y se centra en el aspecto dialógico y accional del lenguaje. Considera la consigna como herramienta mediadora de la actividad de enseñanza, que, a la vez, lo es de la actividad de lenguaje.

En esta perspectiva las actividades de lectura y escritura se analizan en sus procesos particulares de realización. Presenta la articulación de las nociones y el desarrollo de las mismas como objetos de enseñanza.

\footnotetext{
* Propuesta didáctica para la reenseñanza de la lengua formulada para el primer año del nivel universitario.

** Centro Regional Bariloche. Universidad nacional del Comahue. <riestra@bariloche.com.ar>

Sociólogo. Universidad Nacional de Colombia
} 


\section{Summary}

This didactic proposal of re-teaching language is formulated for the first year of the university level. The framework is the historical-cultural approach (Vygotski, Leontiev, Bajtín, Bronckart), centered in the dialogical and actional aspects of the language. It considers the instruction of procedures as a mediating tool of both the teaching activity and, simultaneously, the language activity. In this perspective the activities of reading and writing are analyzed in their particular processes of accomplishment. It presents the articulation of the notions and their development as teaching objects.

\section{La reenseñanza como concepción teóricayla opciónporelinteraccionismo socio-discursivo}

Desde la perspectiva histórico-cultural cada acto de enseñar supone una reenseñanza, en la medida en que al internalizar nuevas acciones y nuevos objetos de conocimiento se produce una reestructuración en las capacidades discursivo-textuales de cada agente. Es el proceso que Vygotski denominó como internalización de las formas culturales.

En este marco se encuadra la programación de la asignatura de primer año del Profesorado en Educación Física Usos y Formas de la lengua escrita. Por consiguiente, no se pretende enseñar lo no enseñado o no aprendido en los otros niveles de enseñanza previos a la universidad, no se trata de llenar huecos de memoria ni huecos de objetos de enseñanza. Sí se trata de interactuar con personas que ingresan a una nueva institución y que necesitan conocer los géneros textuales que circulan en el ámbito universitario para usarlos con eficacia en el desarrollo de la carrera, lo que implica una apropiación instrumental de los mismos.

La concepción del interaccionismo socio-discursivo (Bronckart, 1997), como opción teórica de origen vygotskiano, constituye, específicamente, el referente desde el que han sido seleccionados los contenidos como objetos a ser enseñados. Desde el inicio del dictado de la asignatura en 1997, cuando la propuesta partía de la multidisciplina como opción epistemológica frente a la necesidad de articular objetos de enseñanza provenientes de campos disciplinares de la Ciencias del lenguaje y de la Psicología (Riestra 1998,1999), estos contenidos han ido sufriendo modificaciones.

La posición del interaccionismo socio-discursivo considera al texto como producto de la acción de lenguaje y a este como el instrumento adquirido en el desarrollo histórico. Desde esta visión que se han ido afinando las articulaciones conceptuales de la planificación curricular, puesto que la orientación está dirigida hacia la práctica de lenguaje como una capacidad a desarrollar y, progresivamente, lograr el autocontrol por parte de los alumnos.

Un eje clave en la organización y planificación de los cursos de las acciones de aprendizaje es la direccionalidad en la enseñanza como actividad, entendida esta como acciones con metas y operaciones que las realizan (Leontiev, 1983), perspectiva poco desarrollada en nuestros ámbitos académicos, quizá debido a la influencia piagetiana de los últimos veinticinco años en Argentina.

Por otra parte, cuando me refiero a reenseñar no estoy quitándole relevancia al hecho de que los alumnos ingresantes a la universidad han alcanzado un desarrollo relativamente estabilizado en su capacidad discursivo-textual, y no es este un dato menor, sino todo lo contrario, la situación de los alumnos de primer año nos plantea el desafío comunicativo que consiste en introducir nociones nuevas que deberán articularse y cimentarse en las nociones ya adquiridas por ellos. $Y$ es necesario partir de ese conocimiento del instrumento lenguaje, de la seguridad que da lo conocido (aun cuando se tratare de nociones reduccionistas que operan como obstáculos en la práctica), porque se trata de una habilidad lograda en el desarrollo de una capacidad. 
Lectura y escritura en la universidad: las consignas de tareas en la planificación de la reenseñanza de la lengua

De esta posición se desprende que los tecnicismos solo serán útiles en la medida en que logren ser incorporados como nuevas herramientas, no solo como elementos descriptivos de un estado en un marco teórico. Con esto pretendo aclarar que la solidaridad teórica de las nociones empleadas es importante, siempre y cuando opere en la capacidad de lenguaje, es decir, en la práctica discursivo-textual.

Es este el contexto teórico que ha orientado la organización curricular y los ejes del programa como contenidos a desarrollar en la asignatura (ver Anexo).

\section{Sobre lo accional del lenguaje, el tono bajtiniano y la inversión frente al lenguaje como objeto a ser enseñado}

Bronckart (2001) desde la concepción de la actividad desarrollada por Leontiev y a partir del concepto de acción de Habermas, define la noción de lo accional del lenguaje. Se refiere a lo accional del lenguaje como el juego entre proposiciones y enunciados, juego de interpretaciones posibles e interpretación efectiva. Considera la acción como realidad objetiva y material.

En consonancia con esta noción de acto entre el plano de la lógica y el plano de la valoración subjetiva a que arriba Bronckart, encontramos en Bajtín la referencia a un mundo casi extraobjetual que refleja las relaciones mutuas entre los hablantes. No es el mundo de los tropos, sino de los tonos y matices personales, pero no con respecto a otras personas. Se trata de la relación mutua entre las unidades del discurso. La aguda percepción de lo suyo propio y lo ajeno en la vida discursiva, la importancia excepcional del tono (injuria y alabanza y sus derivados).

El tono es concebido como Separado de los elementos fónicos y semánticos de la palabra y de otros signos. Estos determinan la compleja tonalidad de nuestra conciencia, que sirve de contexto emocional y valorativo durante la comprensión (comprensión completa, comprensión del sentido) del texto leído (o escuchado) por nosotros, así como en una forma más compleja durante la generación creativa de un texto (Bajtín, 1992 p.386)
Si volvemos sobre el concepto de reenseñanza, en nuestra perspectiva didáctica y, teniendo en cuenta la noción de lo accional del lenguaje, reenseñar consiste en reconceptualizar las prácticas ya conceptualizadas por los alumnos universitarios y hacerlo tanto en cuanto acciones que realizan las actividades (lectura y escritura), como respecto de las nociones internalizadas y asumidas como representaciones (en el sentido atribuido por Moscovici y Bourdieu, entre otros).

Debido a la disociación entre el uso del instrumento (lenguaje) y los conocimientos objetivados acerca del mismo (objetos de enseñanza) el enfoque de lo accional puede abrir una perspectiva de carácter instrumental en la didáctica de la lengua.

Para ampliar esta noción del carácter accional del lenguaje es que tomamos la noción de tono que sostiene Bajtín (ob.cit.): El tono no se determina por el contenido objetual del enunciado, ni por los sentimientos y vivencias del hablante, sino por la actitud del hablante respecto de la persona de su interlocutor (su rango, importancia, etc)(Bajtín, 1992: p.377), de carácter social como valoración, pero individual en su realización, no se trata del control racional de la acción, como ha sido desarrollado en la cultura monologista, usando un término bajtiniano, se trata de la emoción, como la percepción del otro (lo ajeno) constitutiva del lenguaje.

El potencial de sentido se sitúa al iniciarse la palabra en un contexto verbal y semántico posible, entre los límites que representan la palabra y la cosa (medio cosístico denomina al que actúa mecánicamente sobre la persona, serían los significados, tanto el general como el del contexto, en su metodología de la comprensión), tanto la palabra como la cosa completan entre ambas el sentido. Descubrir el tono permite transformar la cosa en persona dirá Bajtín.

La interpretación de los sentidos no puede ser científica, pero es profundamente cognoscitiva. 
A su vez, lo accional del lenguaje remite a la realización de la actividad en los términos formulados por Leontiev (ob. cit), a lo extraverbal, la articulación que se realiza a partir de la valoración en la producción del tono social, inherente a lo dialógico del lenguaje como capacidad humana, lo que dicho de otro modo significa que la acción no se origina en la razón, sino en la emoción.

Esta inversión de la concepción dialógica del lenguaje, de origen bajtiniano también se registra en el marco teórico formulado por Maturana (1995), quien, desde otro enfoque epistemológico, define la acción de lenguaje como coordinación de coordinaciones de acciones conductuales, cuya base dialógica se organiza a partir del emocionar entrelazado con el razonar en el lenguajear.

Asimismo, desde la neurología, Damasio (1999) sitúa la relación emoción-pensamiento como continuidad en la prosodia, considerando la entonación una instancia de la prosodia.

Estos enfoques, en relación con el tema específico de la enseñanza de la lengua, abren una perspectiva no ya teórica, sino de orden práctico. Se trata, no solo de considerar los conceptos y nociones desplegados en el discurso de la enseñanza, sino de afinar el análisis en la construcción y el efecto de las acciones de lenguaje que internalizarán los alumnos para alcanzar un nivel de operacionalidad en sus capacidades discursivas y textuales.

Y si la enseñanza de la lengua orientada desde esta perspectiva nos presenta otro objeto a ser enseñado, la noción teórica de acción del lenguaje plantea cuestiones de orden práctico como las siguientes:

a). los alumnos necesitan automatizar determinados aspectos del lenguaje, por lo que es necesario fundamentar el cómo hacerlo.

b). Qué tipo de relación a establecer entre los conceptos provenientes de los mundos formales del conocimiento lingüístico y las prácticas de lenguaje. c). Definir y optar por modelos textuales del intertexto que desarrollarán progresivamente las capacidades textuales de los alumnos.

d). Las acciones de lenguaje de cada enseñante proporcionan (o restringen) nuevos y elaborados instrumentos a la capacidad de lenguaje de los alumnos.

\subsection{El papel de las consignas de tareas: La consigna de trabajo como texto}

Si Bronckart sitúa lo accional del lenguaje entre el nivel proposicional y el plano enunciativo y Bajtín, define al tono a partir de la percepción del otro, encontramos que son nociones confluyentes que abren perspectivas para elaborar instrumentos en nuevos desarrollos de propuestas didácticas.

Como consecuencia, aquí, en esta articulación teórica, la consigna para la enseñanza de la lengua se sitúa como la herramienta fundamental de la planificación. Es necesario definir, en primer lugar, que las consignas de tareas son textos orales y escritos producidos en la interacción socio-discursiva de todos los niveles de enseñanza, cuya coherencia, desde la planificación de las secuencias didácticas, es considerada y analizada como el propio texto (acción de lenguaje) de enseñanza y su posible efecto sobre los alumnos en la realización de las actividades de leer y escribir.

Se trata del texto que media la tarea en la coherencia de su realización, el texto que organiza las acciones mentales en los aprendientes; podemos decir, el que regula, el que ordena, el que dirige, el que prescribe, etc..

En el enfoque de la acción mediada como unidad básica de análisis de las relaciones intermentales (Wertsch, 1999) (interpsicológicas para Vygotski) entre los agentes y la herramienta, ésta es la que llega a configurar la acción de los agentes a pesar de la reflexión consciente y la volición de los propios agentes. La consigna como herramienta cumple esta función de configurar las acciones mentales de los alumnos en la realización de la tarea. 
Lectura y escritura en la universidad: las consignas de tareas en la planificación de la reenseñanza de la lengua

Por eso, al planificar consignas nos referimos a éstas como organizadoras de las tareas, pensamos en las acciones que realizarán, las actividades de lectura y escritura en la complejidad creciente y en relación con las asignaturas específicas de la carrera, que son las que nos ofrecen los géneros textuales utilizados por los alumnos.

De esta manera, la organización de las secuencias didácticas a partir de las consignas que vehiculizan contenidos objetuales y relaciones interpsicológicas a la vez, nos permite situarnos en un espacio diferente de la planificación de la enseñanza de la lengua, el que podrá variar según el nivel, sector social, grupo de aprendientes concretos. Pero esta variación no consiste en dar o dejar de dar contenidos, sino de adaptarlos para interactuar desde el desarrollo potencial en búsquedas de desarrollos próximos.

\subsubsection{La consigna como acción mental}

La consigna que organiza la acción que cada alumno internalizará, puede constituirse en instrumento o bien, puede conducir una acción reproductora y reduccionista, que no favorezca procesos de apropiación de habilidades y de saberes objetivados.

Para que la consigna opere como instrumento de la organización semántica de la conciencia (Vigotski, en Blanck. (1984), el espacio de planificación se conforma como el andamiaje del pensamiento, buscando operar en la zona de desarrollo próximo, a través de las acciones de lenguaje y del metalenguaje que vehiculiza contenidos lingüísticos de las disciplinas de referencia (Ciencias del Lenguaje). La planificación del andamiaje de la consigna supone un plano teórico y un plano práctico, es éste, decididamente, en el que influyen las concepciones teóricas, fundamentalmente las implícitas que circulan entre los enseñantes como material de divulgación (Riestra, 2001).

La planificación desde las consignas se centra en cómo cambia la estructura de la actividad. Se trata del siguiente recorrido: realizar una actividad en primer lugar, después conceptualizarla y a posteriori proponer relaciones posibles con otras actividades y conceptos conocidos para entonces elaborar síntesis significativas. De esta manera, las acciones externas se transforman en acciones mentales internas.

En cambio, la consigna que demanda la reproducción de un concepto o la relación entre una o más nociones o la aplicación de las mismas, produce una reducción de significado, en el mejor de los casos, una operación de memoria funcional, no exigirá una actividad de reorganización semántica en la conciencia de los alumnos.

Estos problemas, por lo general difusos en la enseñan$\mathrm{za}$, tienen causas diversas y complejas que han sido objeto de análisis (Bronckart, 1985), aunque en la actualidad, en general, para enseñar lengua se recorren varias disciplinas de las Ilamadas ciencias del lenguaje y se toman las conceptualizaciones más novedosas, sin que medien transposiciones didácticas. Debido a esta situación los alumnos deben analizar con categorías que van desde la narratología a la lingüística textual, pasando, en algunos casos por la gramática, se trata de categorías que no se transforman operacionalmente en instrumentos de comprensión y producción de textos.

Sin negar la utilidad de la taxonomía, es necesario buscar la inserción del concepto enseñado de manera descontextualizada, en las acciones de lenguaje. La clasificación tiene utilidad cuando está apoyada en la acción para modificar el comportamiento de lenguaje. A modo de ejemplo podemos ver que, mientras los análisis gramaticales fueron de una complejidad media tuvieron una utilidad relativa en el proceso de aprendizaje de la actividad de lenguaje, fundamentalmente de los tipos de discurso. Servían para cotejar el propio texto producido con el modelo analizado. En la medida en que fueron complejizándose los análisis y exigiendo mayor abstracción (en función de los modelos teóricos implicados) -siempre nos referimos al ámbito escolar en sus diferentes niveles- fueron per- 
diendo la utilidad o, desde nuestro marco, podemos decir que no pudieron internalizarse, se mantuvieron como objetos ajenos a la práctica discursiva y ocuparon el espacio vital del aprendizaje de los alumnos sin los resultados esperados por los docentes.

\subsubsection{La propuesta didáctica: Lo accional del lenguaje se realiza en lo dialógico}

Como la situación y el auditorio son los elementos determinantes de la enunciación, el sentido depende del otro social que, como parte extraverbal sobreentendida, realizará la comunicación o interacción verbal, tanto en los géneros cotidianos (primarios) como en los secundarios.(Bajtín, ob.cit.)

A partir de esta concepción bajtiniana de los géneros discursivos, la noción de género textual que adopta Bronckart (1997), en tanto textos adaptados a las opciones de las formaciones socio-lenguajeras como modelos disponibles en un intertexto, es un concepto instrumental, la producción verbal situada comunicativamente con un determinado uso social que va modificándose históricamente en el proceso intergeneracional.

Schneuwly (1995) ha analizado el género como megaherramienta, por cuanto es una configuración estabilizada de muchas herramientas y muchos subsistemas semióticos que sirven para actuar con eficacia en determinadas situaciones comunicativas.

Desde este enfoque el concepto de género textual es el que inicia el trayecto de reenseñanza propuesto y es, a la vez, el que estructura las secuencias didácticas en una progresión de complejidad creciente de cuatro géneros elegidos: la reseña, la entrevista, el informe, el artículo de opinión, la monografía y el ensayo. La decisión de adoptar estos géneros y no otros proviene del uso relativamente estable que tienen en el ámbito académico universitario.

\subsubsection{El trayecto didáctico de USO/ SENTIDO/ FORMA}

en las actividades de leer y escribir ..La comprensión dialógica activa y la actividad de lectura

En la realización de la comprensión dialógica formulada por Bajtín se plantea la relación texto-contexto: Cada palabra del texto conduce fuera de sus límites. Toda comprensión representa la confrontación de un texto con otros textos. (Bajtín, 1992: p.383)

El momento valorativo de la comprensión es previo a la racionalización del sentido, que solo es relativa. El sentido es el contacto entre personas y por lo tanto la lectura es enfocada como una actividad que se realiza a través de acciones y operaciones (Leontiev, 1983).

Lo activo consiste en que la comprensión es confrontación con otros textos y en un contexto nuevo, es decir el carácter dialógico de la comprensión consiste en la percepción de su dinámica, el propio contexto, el contexto del autor.

En la actividad de lectura la noción utilizada como soporte es la de contexto de producción (físico, social y subjetivo) (Bronckart, 1997), determinante del sentido que deberá elaborar el lector.

Las consignas orientan las acciones de: 1) búsqueda del tema del texto, 2) posición del autor respecto del tema, 3) resumen del contenido y 4)opinión respecto de la relación entre tema y posición del autor.

El trayecto didáctico parte del uso (contexto de producción), se detiene en el sentido (tema y posición del autor) y analiza la forma (niveles de construcción textual)

El modelo de arquitectura textual del interaccionismo socio-discursivo (Bronckart, ob.cit) contempla los siguientes niveles de análisis: 
Lectura y escritura en la universidad: las consignas de tareas en la planificación de la reenseñanza de la lengua

1. Infraestructura: plan general del texto, tipos de discurso, secuencias textuales.

2. Mecanismos de textualización: conexión, cohesión nominal, cohesión verbal

3. Mecanismos de enunciación: posición enunciativa, voces, modalizaciones.

Este modelo teórico es abordado desde el trayecto didáctico (uso- sentido-forma), que permite incorporar los niveles en progresión de complejidad y dentro de las dos actividades que se distinguen específicamente en su ejecución durante el desarrollo del curso: la lectura y la escritura. La diferenciación tiene un carácter teórico y práctico, en la medida en que se articulan nociones diversas y capacidades también diversas.

La actividad de lectura tiene, por lo general, un desarrollo más rápido que la actividad de escritura, lo que pone de manifiesto que el análisis de la forma de los textos en la actividad de lectura, no produce un conocimiento y una apropiación inmediata de la capacidad discursivo-textual en la actividad de escritura, por lo que se trabajan como procesos diferenciados.

La construcción del sentido y lo accional en la actividad de escritura

Para la construcción del sentido del texto escrito el concepto del otro como voz internalizada que orienta socialmente la producción verbal entre los individuos vinculados por ciertas condiciones comunes de la vida conforma el contexto extratextual determinante de todo texto.

Por una parte, el fondo dialogizador de la percepción en Bajtín, y lo accional del lenguaje en Bronckart (2001), concebido como construcción en sincronía con la realización del hombre (operaciones textuales) dentro, en y a través, de un texto singular son dos nociones teóricas que confluyen para construir la noción de texto como textualización, la acción de textualizar. En los textos escritos que nos ocupan las decisiones simultáneas y sucesivas de la operacionalización integran tanto tipologías discursivas como secuencias textuales, deteniéndose en las relaciones anafóricas, que provienen del plan general del texto y se construyen internamente desde el tema que se despliega.

Dentro del trayecto didáctico, los mecanismos de enunciación son decisiones (operaciones) controladas desde el uso (voces y modalizaciones) y el sentido (tipos de discurso y secuencias textuales), mientras que los mecanismos de textualización son decisiones y evaluaciones realizadas desde la forma (conectores, cohesión nominal y verbal).

Resta agregar un punto de la propuesta didáctica que hace a la coherencia de este constructo o aparato de enseñanza, la escala de evaluación, instrumento que es presentado al inicio del curso y va cargándose de significado durante el desarrollo, con la finalidad de que los alumnos se apropien del mismo para ejercer el autocontrol que se propone como objetivo de la asignatura:

\section{1) Escala de evaluación}

2) lenguaje adecuado a la situación comunicativa

3) coherencia y jerarquización de la información

4) puntuación

5) sintaxis

6) precisión léxica

7) ortografía

8) presentación y legibilidad

Pese a que la mayoría de los alumnos desconoce el significado y alcance de las nociones presentadas en la escala, a medida que van internalizando y reconociendo las conceptualizaciones de las actividades que realizan, la jerarquización cobra sentido, de acuerdo con el nivel de desarrollo que cada uno va logrando en sus habilidades. De esta manera, la concepción de lenguaje como capacidad y como acción va sustituyendo la concepción de lengua como saber abstracto y ajeno, con la que inician el cursado de la materia.

Cuando cada alumno hace uso personal de la escala, particularmente en la actividad de escritura, la 
utilidad de la misma se introduce como término de negociación respecto de los avances en el desarrollo y las dificultades que aún deben superar. Podemos decir que la escala de evaluación toma forma como instrumento compartido entre quienes enseñamos y quienes aprenden.

3. La actividad de enseñanza de las capacidades de lenguaje

A modo de resumen, el énfasis puesto en las consignas se origina en la paradojal situación que nos presenta la enseñanza de la lengua: las nociones movilizadas al enseñar pueden comprenderse pero no producen, necesariamente, el efecto de dominio de la capacidad de leer o escribir. Esto nos plantea un problema de orden práctico, cuya investigación requiere préstamos no sólo de las denominadas ciencias del lenguaje, sino del campo de la psicología y de las ciencias humanas y sociales en general.

Así como la actividad objetal genera el movimiento interno de la conciencia individual, que a su vez es un sistema dual con un significado fijado en el lenguaje y un sentido como significado personal (Leontiev, 1983.), la consigna de tareas se constituye como instrumento mediador que dirige desde el plano de la acción de lenguaje y controla las operaciones mentales que se incorporan para realizar la actividad.

Tanto en la actividad de lectura, por una parte, como en la planificación de la escritura, por otra, se trata de proponer las tareas cuyas metas van incorporando en forma secuencial los saberes instrumentales incorporados en cada género textual.

La diferencia entre enseñar a partir de capacidades cognitivas abstractas y enseñar a partir de objetos socialmente construidos (textos que genéricamente circulan en los ámbitos académicos universitarios) y acciones cuyas metas apuntan a apropiarse del objeto operacionalmente, o lo que es lo mismo, apropiarse de la capacidad a desarrollar en la realización de la actividad concreta, estriba en considerar o no al lenguaje como comportamiento.
En lo expuesto y pese a la síntesis, quizá demasiado apretada de este artículo, he buscado destacar el eje de la propuesta, la concepción del lenguaje como comportamiento que, dicho de otro modo, es enfocar el lenguaje en cuanto acción humana, la acción que media todas las otras en sus realizaciones de las diversas actividades que vamos desarrollando los humanos a lo largo de la historia.

Pero es la práctica la que pone la medida de eficacia y coherencia de una propuesta didáctica $y$, entre los logros y obstáculos que se presentan, habrá que reelaborar a nivel teórico un nuevo problema y reanudar la búsqueda de nuevas propuestas.

Anexo: Programa de Usos y Formas de la Lengua Escrita

Objetivo: Capacitar a los alumnos en la lectura y escritura de textos en el ámbito universitario).

\section{Autoevaluación. La producción de los textos escritos. Contexto. Paratexto.}

- El género textual como instrumento

- Articulación USO-SENTIDO-FORMA

-El uso y la forma: técnicas adquiridas. La apropiación como proceso.

- Las actividades de lectura y escritura. El resumen.

- Paráfrasis. Ortografía: acentuación

\section{Lectura: texto de instrucción/reglamento.}

- Gramática. Las relaciones morfológicas y sintácticas: verbos/clases de palabras.

- La oposición sustantivo-verbo.

- Secuencias prototípicas de base textual.

- Empleo de tiempos verbales

- Uso: guiar la acción - reglar la acción

-Escritura: textos breves: Relato. Opinión. Explicación. Descripción.

-Instrucciones. Reglamento 
Lectura y escritura en la universidad: las consignas de tareas en la planificación de la reenseñanza de la lengua

\section{Lectura: entrevista. Volumen completo.}

- Niveles de análisis textual: tema (macroestructura semántica, macrorreglas)

- La técnica del mapa conceptual.

- Forma: -Marcas lingüísticas de la enunciación indirecta.

-Nexos coordinantes y subordinantes. Puntuación.

-Uso: Sujetos discursivos.

-Escritura: Texto referido. Reseña.

\section{Lectura: Informe}

-Exposición. Mundos discursivos-tipos de discurso

-Elaboración de los núcleos semánticos en la lectura.

- Plan del texto.

- Forma: progresión temática. Relaciones anafóricas.

- Uso: Fórmulas de cada género textual. Ámbito de producción

-Escritura: Informe. Monografía

\section{Lectura: Ensayo}

-Núcleos semánticos. Argumentación

- Forma: - posición enunciativa. Voces y modalizaciones

-Uso: tipos de discurso

- Escritura: Artículo de opinión. Ensayo.

\section{Referencias}

Adam, J.-M.(1992) Les textes, types et prototypes. Paris: Nathan

Bajtín, M. (1992) Estética de la creación verbal. México: Siglo XXI

Blanck, G. (1984) Vygotski: memoria y vigencia. Buenos Aires: Cognición
Bronckart, J.-P. (1985) Las ciencias del lenguaje: ¿un desafío para la enseñanza?. Paris: UNESCO

Bronckart, J.-P.(1997) Activité langagière. Textes et discours. Paris: Delachaux et Niestlé

Bronckart, J.-P.(2001) Les types des discours comme traces cristallisées de l'action du langage ».

- Les analyses de discours au défi d'un dialogue romanesque. Nancy : E. Roulet (Ed) PUN.

Damasio, A. (1999) Sentir lo que sucede. Cuerpo y emoción en la fábrica de la consciencia. Santiago de Chile : Ed. Andrés Bello

Leontiev, A.(1983) El desarrollo del psiquismo. Madrid: Akal

Maturana, H. (1995) La realidad: ¿objetiva o construida?. Barcelona: Anthropos

Riestra, D. (1999) "Reenseñar la escritura a estudiantes universitarios". Infancia \& Aprendizaje. Na 88, 43-56.

Riestra, D. (2001, en prensa) "Los productos de la transposición didáctica en la enseñanza de la lengua: ¿instrumentos u obstáculos?". Actas de las 2as Jornadas de Lingüística aplicada a la enseñanza de la lengua. Comahue : Universidad. Nacional

Schneuwly, B. (1995) Apprendre à écrire. Une approche socio-historique en Vers un modèle d'enseignement de l'écriture. Québec: Les editions logiques

Vigotsky, L.(1996).El desarrollo de los procesos psicológicos superiores. Barcelona: Crítica

Wertsch, J. (1999) La mente en acción. Barcelona: Crítica. Buenos Aires:Aique (ED.). 